\title{
Safety of opioid patch initiation in Australian residential aged care
}

$\mathrm{O}$ pioid analgesics are recommended for the treatment of cancer pain and for shortterm treatment of moderate to severe acute pain. ${ }^{1,2}$ The number of opioid prescriptions in Australia reimbursed by the Pharmaceutical Benefits Scheme increased from 2.4 million in 2002 to 7.0 million in $2007 .^{3}$ Much of this increase is probably driven by the increased administration of opioids for chronic non-cancer pain, ${ }^{3,4}$ but such use remains controversial, especially in older people, as they are more susceptible to adverse drug events. ${ }^{5-7}$

Transdermal opioid patches have been designed to provide long-lasting therapy for patients with chronic pain. Two opioids are available for administration via transdermal patches: fentanyl and buprenorphine. Fentanyl patches have a duration of action of 3 days, while buprenorphine patches are active for 7 days. ${ }^{1}$ In addition to an extended duration of action, transdermal opioids also have a slower onset of action, making them unsuitable for the management of acute pain. $^{1}$

For chronic, non-cancer pain, Australian guidelines recommend a stepwise approach, with an initial trial of nonopioid analgesics followed by weak opioids if simple analgesics are not effective. ${ }^{8,9}$ Oral morphine or oxycodone and transdermal buprenorphine are considered first-line options for chronic non-cancer pain. For frail or older patients, a low dose of immediate-release opioid may be used to assess responsiveness; $;, 9$ once initial dosing requirements have been determined, sustained-release preparations, including transdermal buprenorphine patches, may increase patient compliance, as the frequency of administration is reduced compared with other dosing forms. 7,10

Potent opioids, such as fentanyl, are only recommended for patients with chronic severe and disabling pain who exhibit tolerance for opioids and

\section{Abstract}

Objective: To explore opioid use by aged care facility residents before and after initiation of transdermal opioid patches.

Design: A cross-sectional cohort study, analysing pharmacy data on individual patient supply between 1 July 2008 and 30 September 2013.

Setting: Sixty residential aged care facilities in New South Wales.

Participants: Residents receiving an initial opioid patch during the study period.

Main outcome measure: The proportion of residents who were opioidnaive in the 4 weeks prior to patch initiation was determined. In addition, the patch strength at initiation and the daily dose of transdermal patches and of additional opioids 1 month after initiation were determined.

Results: An opioid patch was initiated in 596 of 5297 residents (11.3\%: $2.6 \%$ fentanyl, $8.7 \%$ buprenorphine) in the 60 residential aged care facilities. The mean age at initiation was 87 years, and $74 \%$ of the recipients were women. The proportion of recipients who were opioid-naive before patch initiation was 34\% for fentanyl and 49\% for buprenorphine. Most were initiated at the lowest available patch strength, and the dose was up-titrated after initiation. Around 15\% of fentanyl users and 10\% of buprenorphine users needed additional regular opioids after patch initiation.

Conclusions: The results suggest some inappropriate initiation of opioid patches in Australian residential aged care facilities. Contrary to best practice, a third of residents initiated on fentanyl patches were opioidnaive in the 4 weeks before initiation.

do not respond to non-narcotic analgesics. ${ }^{1,10,11}$ The margin between the therapeutic and toxic doses of fentanyl is small, and it has been associated with a number of fatalities in opioid-naive patients. ${ }^{12,13}$ Guidelines recommend that fentanyl is most appropriate in the treatment of opioid-tolerant palliative care patients and patients with cancer. ${ }^{8}$ Fentanyl patches should not be used in opioidnaive patients, and the equianalgesic opioid dose should be determined before initiation of the patch. ${ }^{8,11}$

Given the difficulties with the dose titration of transdermal buprenorphine preparations and concerns about the safety of fentanyl in opioidnaive patients, the use of transdermal preparations in older patients should be limited to opioid-tolerant patients with stable opioid requirements.

The aim of our study was to explore analgesic use before and after the initiation of transdermal opioid patches in residents of Australian aged care facilities. The specific objectives were to determine the proportion of people who were opioid-naive or opioid-tolerant prior to initiation of the transdermal product, and to determine how initiation of transdermal opioids affected the use of other opioids.

\section{Methods}

\section{Data source}

De-identified pharmacy data on all medicines supplied in dose administration aids to 60 residential aged care facilities in New South Wales during the period 1 July 2008 to 30 September 2013 were analysed. All medications supplied to residents in these facilities, including over-thecounter products, must be packed in a dose administration aid that comprises blisters containing all medications administered at a single dosing point, enabling assessment of intended co-administration. The blisters 
also reflect any changes made to any medicine in the weekly supply, so that all changes are identified at the time they occur.

The database therefore provides complete medication records for all residents, including a unique resident identifier, sex, date of birth, date of death, generic and brand medication names, duration for which each medicine was dispensed, strength, dose and dosage instructions for each medicine, and pro re nata use ("as needed").

\section{Analgesic medicines included in the analyses}

Included in the analyses were narcotic analgesics (opioids: codeine, codeine with paracetamol, methadone, morphine, hydromorphone, oxycodone, tramadol, pethidine, dextro-propoxyphene, fentanyl and buprenorphine), simple analgesics (paracetamol) and oral non-steroidal anti-inflammatory drugs (NSAIDs: including COX-2 selective inhibitors, but excluding topical preparations).

\section{Patch initiation and previous analgesic use}

A cross-sectional cohort study was undertaken to determine the use of analgesics in the 4 weeks prior to initiation of fentanyl and buprenorphine transdermal patches. Incident opioid patch use was defined as the first (index) dispensing of a fentanyl or buprenorphine patch between 1 July 2008 and 30 September 2013, with no use during the previous year. Patients who received their index opioid patch at the time of their admission to the residential aged care facility were excluded, because their analgesic use in the previous year could not be established. The proportions of residents initiated on patches who were naive to all analgesics or to opioids and those who were opioid-tolerant were determined. Opioid-naive users were defined as those who had not received an opioid analgesic (but may have received other simple or NSAID analgesics) in the 4 weeks prior to their first patch. Opioidtolerant residents were those who had received an opioid, either regularly or pro re nata, in the 4-week period before patch initiation. The initiation strength of the patches was also determined.

\section{Patch initiation and opioid use}

The proportion of residents starting on a fentanyl or buprenorphine patch who received opioids (regularly or pro re nata) during the 30 days before or after patch initiation was calculated as a percentage of the entire sample of patch initiators.

Daily doses of regular opioids other than patches, when used concurrently with the patches, were calculated for the 30 days before and the 30 days after patch initiation. The dose of each generic agent in combination products was determined separately. The daily dose of each medicine on a given day was calculated from the product of the strength of the medicine and the dosage instructions (eg, oxycodone $10 \mathrm{mg}$ twice a day = daily dose of $20 \mathrm{mg}$ ). Opioid strengths, including transdermal patches, were converted to oral morphine equivalents using opioid equianalgesic conversion ratios. ${ }^{14}$ Transdermal fentanyl was converted to the lowest daily oral morphine dose in the range provided (ie, $12 \mu \mathrm{g} / \mathrm{h}$ fentanyl to $30 \mathrm{mg}$ morphine, and $25 \mu \mathrm{g} / \mathrm{h}$ fentanyl to 60 $\mathrm{mg}$ morphine).

The mean daily dose was calculated for each patch and for the opioids in total. For the dose calculation, asneeded use was excluded because of the lack of information about the actual daily usage.

\section{Statistical analyses}

The Pearson $\chi^{2}$ test and the Student $t$ test were applied to compare cohort

\begin{tabular}{|lccc|}
\hline $\begin{array}{l}1 \text { Characteristics of aged care residents in whom transdermal opioid } \\
\text { patches were initiated }\end{array}$ & $\begin{array}{c}\text { Fentanyl } \\
\text { initiators }\end{array}$ & $\begin{array}{c}\text { Buprenorphine } \\
\text { initiators }\end{array}$ & $P$ \\
\hline Number & 137 & 459 & \\
Mean age at initiation, years (SD) & $86(8.4)$ & $87(7.6)$ & $0.148^{*}$ \\
Sex & & & \\
Male & $30(22 \%)$ & $126(27 \%)$ & $0.194^{\dagger}$ \\
Female & $107(78 \%)$ & $333(73 \%)$ & \\
\hline$*$ Student $t$ test. $+\chi^{2}$ test. & & & \\
\hline
\end{tabular}

characteristics and proportions. Linear regression trend lines were fitted to the trends in daily dose after initiation of the patches. Analyses were performed with the SAS 9.4 statistical package (SAS Institute).

\section{Ethics}

This study was approved by the Sydney Local Health District Human Research Ethics Committee, Concord Repatriation General Hospital (CH62) 6/2010-49 HREC/10/CGRH/57).

\section{Results}

\section{Patch initiation and previous analgesic use}

The dataset included 5297 residents who received at least one medication between 1 July 2008 and 30 September 2013. Fentanyl patches were initiated in 137 individuals (2.6\%) $(8.7 \%)$. The mean age was similar in both treatment groups, and $74 \%$ were women (Box 1).

More than a third $(34 \%, 46$ of 137$)$ of residents who began using a fentanyl patch and almost half $(49 \%, 224 / 459)$ of those who began using a buprenorphine patch were opioid-naive (Box 2).

Of the opioid- or analgesic-naive residents who received a fentanyl or buprenorphine patch, $87 \%$ (46 of 53) and $92 \%$ (229 of 250), respectively, commenced on the lowest available strength $(12 \mu \mathrm{g} / \mathrm{h}$ fentanyl; $5 \mu \mathrm{g} / \mathrm{h}$ buprenorphine). Further, more than $80 \%$ of those who had received opioids before a transdermal patch were also started on the lowest strength and buprenorphine patches in 459 


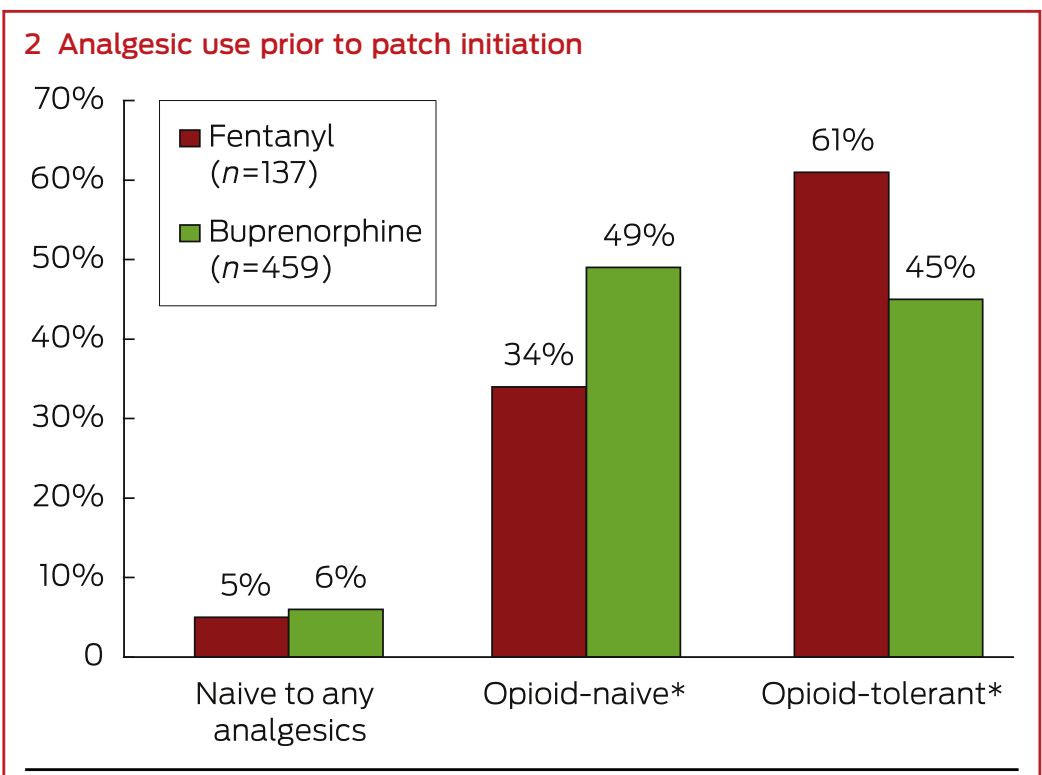

$* P<0.05$ for difference between fentanyl and buprenorphine initiators ( $\chi^{2}$ test).

dose (69 of 84 for fentanyl; 168 of 209 for buprenorphine). No opioid- or analgesic-naive residents who received a fentanyl patch were initiated on the highest strength $(50 \mu \mathrm{g} / \mathrm{h})$, and only three of the 250 opioid- or analgesic-naive patients who received a buprenorphine patch were initiated on the highest strength $(20 \mu \mathrm{g} / \mathrm{h})$.

\section{Patch initiation and opioid use}

After patch initiation, residents receiving fentanyl and buprenorphine were up-titrated to higher doses (Box 3).

Just under $30 \%$ of fentanyl initiators had used regular opioids (Box 4) and just under $25 \%$ had been using asneeded opioids in the 4 weeks before patch initiation (Box 5). About $20 \%$ of buprenorphine initiators had previously used regular opioids (Box 4) and about $18 \%$ had received them as needed (Box 5). Most residents were receiving oral formulations. The most commonly used opioids before patch initiation were oxycodone and codeine.

After patch initiation, the proportion of those who continued to receive regular opioids concurrently with their patch was about $15 \%$ (21 of 137) for fentanyl users and $10 \%$ (47 of 459 ) for buprenorphine users (Box 4). In residents by day 30 to $30 \%$ (41 of 137 ) for fentanyl and to $23 \%$ (106 of 459) for buprenorphine (Box 5).

\section{Discussion}

The availability of transdermal opioid formulations provides increased analgesic options for the management of chronic pain. However, initiation of transdermal opioids requires additional caution with the appropriate choice of agent and dose titration, especially in older patients. Our results indicate that fentanyl patches were initiated in $2.6 \%$ of aged care residents, and buprenorphine patches in $8.7 \%$. While the use of transdermal buprenorphine is recommended for the management of chronic pain, when commencing opioids in older patients a low-dose immediaterelease preparation is recommended once their opioid requirements have been ascertained, and the dose should then be titrated accordingly. ${ }^{8,9}$ Transdermal fentanyl is not recommended in opioid-naive individuals because of its high potency. In this study, a relatively high use of both fentanyl and buprenorphine patches in opioid-naive aged care residents was observed, and this raises safety concerns.

Fentanyl is a highly potent opioid. It is not appropriate for opioid-naive needed increased immediately after patch initiation, but then decreased
3 Daily dose of fentanyl or buprenorphine, presented as morphine equivalents*

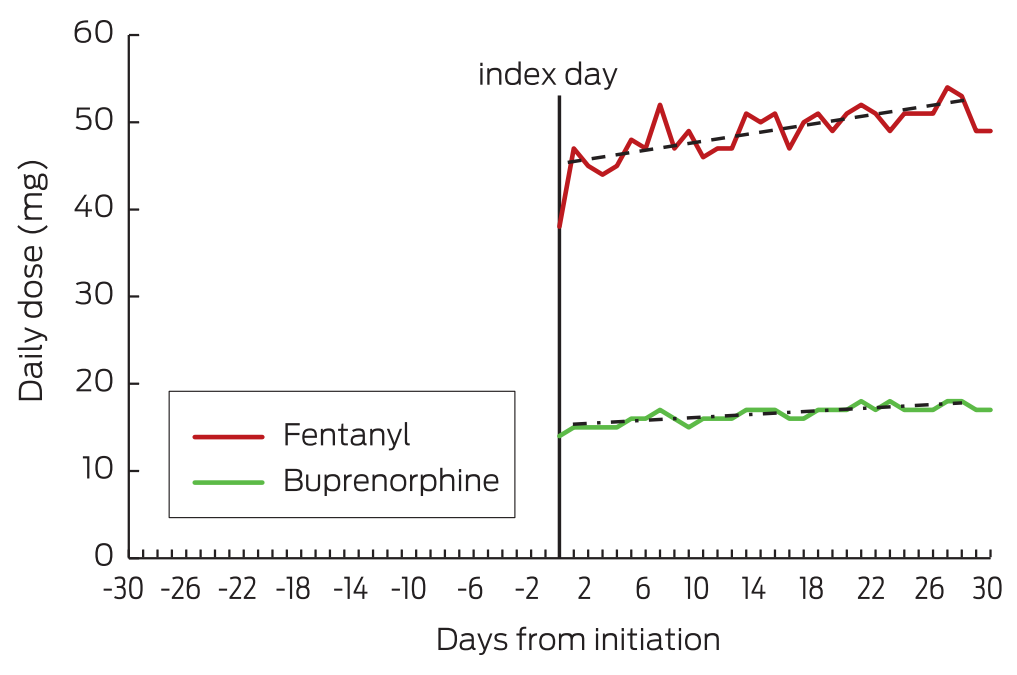

* As-needed opioids were excluded from analysis because of the lack of information on the dose administered. 
4 Proportion of residents receiving regular opioids before and after patch initiation*

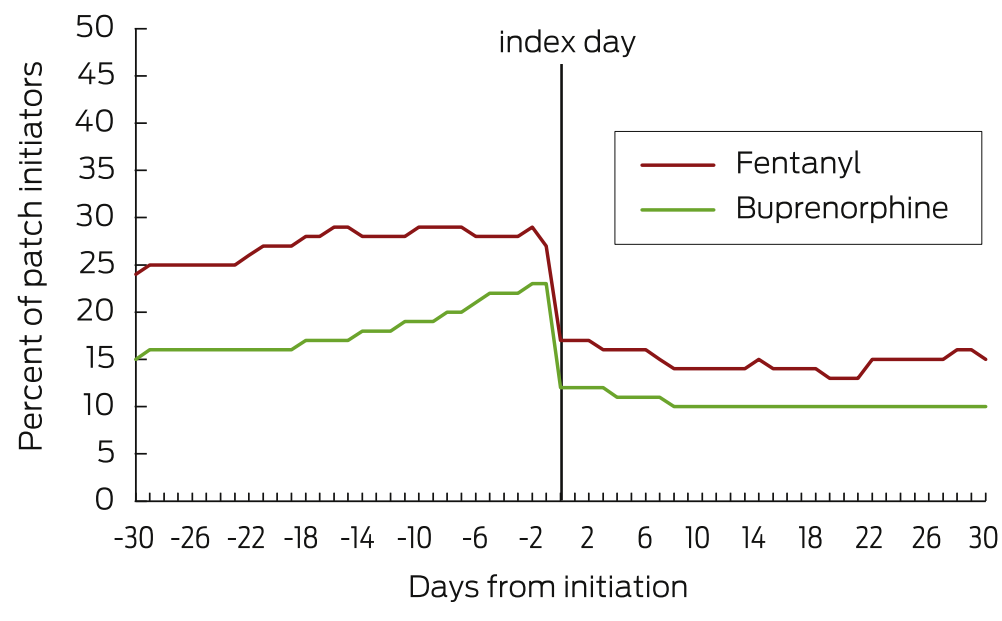

* After-patch initiation use denotes regular opioid use concurrent with transdermal patch use.

patients because of the risk of toxicity, including respiratory depression and overdose-related mortality. ${ }^{12,13}$ The United States Food and Drug Administration released a number of safety communications regarding the dangers of fentanyl patches in opioid-naive patients. ${ }^{15}$ In Australia, the Pharmaceutical Benefits Scheme allows a restricted benefit for the treatment of chronic severe disabling pain that does not respond to non-narcotic analgesics. In our study, a third of aged care residents who were commenced on a

5 Proportion of residents receiving as-needed opioids before and after patch initiation*

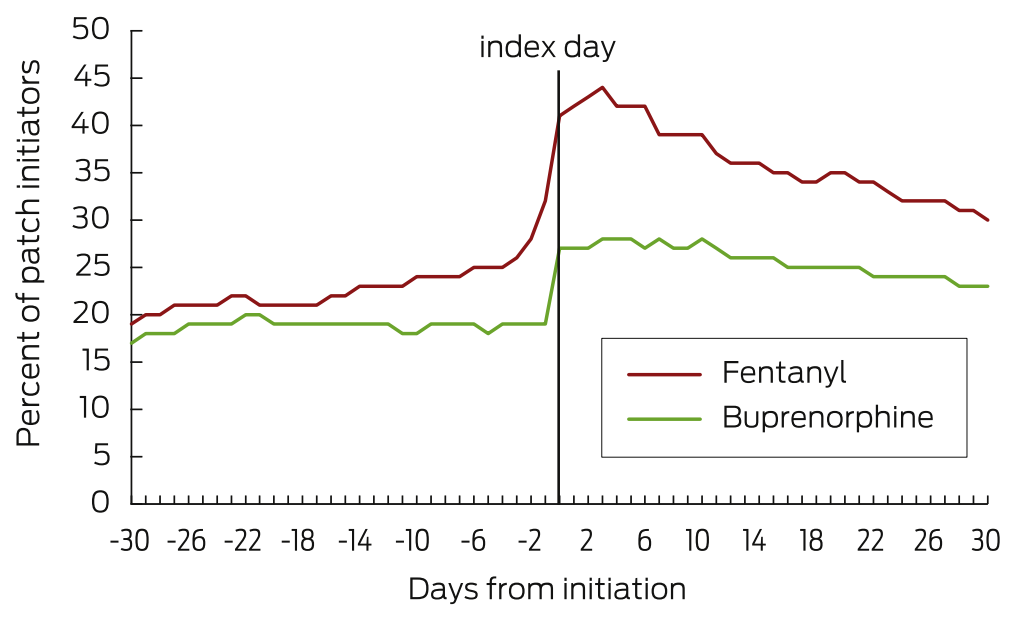

* After-patch initiation use denotes as-needed opioid use concurrent with transdermal patch use. fentanyl patch were opioid-naive. Our findings in residents who had previously used opioids are similar to those of a Dutch study which reported that $60 \%$ of patients who began using fentanyl patches had previously used other opioids. ${ }^{16}$ If fentanyl patches are to be used in opioid-naive patients with cancer pain, they should not be initiated at doses greater than $25 \mu \mathrm{g} / \mathrm{h}^{11} \mathrm{We}$ found that no opioid- or analgesicnaive patients were initiated with fentanyl patches at doses higher than this. 


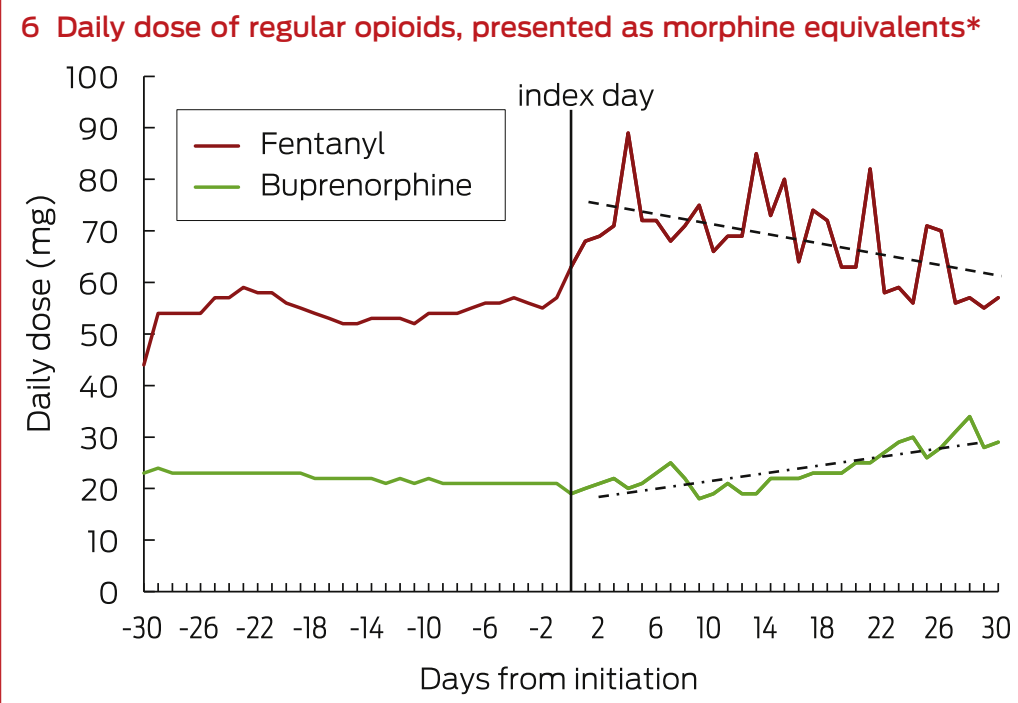

* After-patch initiation use denotes as-needed opioid use concurrent with transdermal patch use. As-needed opioids have been excluded from analysis because of the lack of information on administered dose.

chronic pain. Guidelines indicate that if transdermal opioid patches are initiated, additional, immediaterelease analgesics may be codispensed in the case of breakthrough pain. ${ }^{1}$ Non-opioid analgesic options may be considered in people without cancer, while short-action opioids should be used as needed in those with cancer. ${ }^{2,10}$ Even though opioid use decreased after patch initiation, we found that $15 \%$ of fentanyl users and $10 \%$ of buprenorphine users were still taking regular opioids concurrently with their patches. This is similar to a postmarketing surveillance study which reported that $14 \%$ of patients needed concomitant opioids after starting buprenorphine therapy. ${ }^{19}$ We found that the required doses of regular concurrent opioids decreased after patch initiation in those receiving fentanyl, while they increased in those receiving buprenorphine. Further, a third of fentanyl users and a quarter of buprenorphine users received concomitant oral or parenteral opioids on an as-needed basis 1 month after patch initiation.

\section{Study strengths and limitations}

The dataset used in this study provided a complete medication history for each resident in 60 aged care facil- ities. All medications, including those purchased over the counter, are captured in the dataset. This is important, as many paracetamol and NSAID preparations are available over the counter in Australia. While a complete medication history was available for each resident, the dataset does not contain any clinical information, such as indication, so that it is possible that some of the analgesics were used for acute or cancer pain, or for palliative care. Problems of safety and adequate pain control in opioid-naive patients are not related to the indication for which they are taken, so that the absence of data on indication is unlikely to affect our results. A second limitation was the lack of dosing information for as-needed medicines. As-needed opioids were included in all our analyses, except when calculating the total daily opioid dose.

\section{Conclusion}

Our results suggest a certain degree of inappropriate initiation of opioid patches in Australian residential aged care. Contrary to best practice, a third of residents commencing fentanyl patches and almost half of those commencing buprenorphine patches were opioid-naive in the 4 weeks before initiation of the transdermal patch.

Acknowledgements: We acknowledge Mr Gerard Stevens, managing director of Webstercare, for his contribution to data collection and provision.

Competing interests: No relevant disclosures.

(c) 2015 AMPCo Pty Ltd. Produced with Elsevier B.V. All rights reserved. 
1 Pharmaceutical Society of Australia, Australasian College of General Practitioners, Australasian Society of Clinical and Experimental Pharmacologists and Toxicologists. Australian medicines handbook 2010. Adelaide: AMH, 2010.

2 Macintyre PE, Scott DA, Schug SA, et al. Acute pain management: scientific evidence. 3rd edition. Melbourne: Australian and New Zealand College of Anaesthetists and Faculty of Pain Medicine, 2010. http:// sydney.edu.au/medicine/pmri/pdf/ Acute-pain-management-scientificevidence-third-edition.pdf (accessed Jul 2015).

3 Leong M, Murnion B, Haber P. Examination of opioid prescribing in Australia from 1992 to 2007. Intern Med J 2009; 39: 676-681.

4 Boudreau D, Von Korff M, Rutter C, et al. Trends in long-term opioid therapy for chronic non-cancer pain. Pharmacoepidemiol Drug Saf 2009; 18: 1166-1675.

5 Van Ojik A, Jansen P, Brouwers J, et al. Treatment of chronic pain in older people: evidence-based choice of strong-acting opioids. Drugs Aging 2012; 29: 615-625.

6 Gomes T, Mamdani M, Dhalla I, et al. Opioid dose and drug-related mortality in patients with nonmalignant pain. Arch Intern Med 2011; 171: 686-691.

7 Pergolizzi J, Böger RH, Budd K, et al. Opioids and the management of chronic severe pain in the elderly: consensus statement of an International Expert Panel with focus on the six clinically most often used World Health Organization Step III opioids (buprenorphine, fentanyl, hydromorphone, methadone, morphine, oxycodone). Pain Pract 2008; 8: 287-313.

8 Analgesic Expert Group. Therapeutic guidelines: analgesics. Version 5. Melbourne: Therapeutic Guidelines, 2007.

9 Royal Australian College of Physicians. Prescription opioid policy: improving management of chronic non-malignant pain and prevention of problems associated with prescription opioid use. Sydney: RACP, 2008. http://www.fpm. anzca.edu.au/resources/professionaldocuments/documents/Prescription\% 200pioid\%20Policy.pdf (accessed Jul 2015).

10 NPS MedicineWise. Opioids - a planned approach to prescribing opioids for persistent non-cancer pain. MedicineWise News, 1 Jun 2010. http:// www.nps.org.au/publications/healthprofessional/nps-news/2010/npsnews-69 (accessed Jul 2015).

$1 \quad$ NPS MedicineWise. Generic brand of fentanyl patches (Denpax) PBS listed. NPS RADAR, 1 Aug 2011. http://www. nps.org.au/publications/healthprofessional/nps-radar/2011/august2011/brief-item-generic-fentanylpatches (accessed Jul 2015).

12 Paparella S. A serious threat to patient safety: the unintended misuse of fentanyl patches. J Emerg Nurs 2013; 39: 245-247.

13 Grissinger K. Inappropriate prescribing of fentanyl patches is still causing alarming safety problems. P\&T 2010; 35: 653-654. http://www.ncbi.nlm.nih. gov/pmc/articles/PMC3008378/ (accessed Jul 2015).

14 Government of Western Australia, Department of Health. Opioid conversion guide. http://www. healthnetworks.health.wa.gov.au/
cancer/docs/Opioid_Conversion.pdf (accessed Jul 2015).

15 US Food and Drug Administration. FDA requiring colour changes to Duragesic (fentanyl) pain patches to aid safety emphasizing that accidental exposure to used patches can cause death [media release]. Sep 2013. http://www. fda.gov/Drugs/DrugSafety/ucm368 902.htm (accessed Jul 2015).

16 Breekveldt-Postma N, Penning-van Beest F, Hering R. Utilisation pattern of fentanyl transdermal system in the Netherlands. Pharmacoepidemiol Drug Saf 2005; 14: 129-134.

17 Ball A, Smith K. Optimizing transdermal drug therapy. Am J Health Syst Pharm 2008; 65: 1337-1346.

18 Pergolizzi J, Ben-Joseph R, Chang C, et al. US practitioner prescribing practices and patient characteristics of those newly treated with a buprenorphine transdermal patch system. Curr Med Res Opin 2014; 30: 1579-1587.

19 Griessinger N, Sittl R, Likar R. Transdermal buprenorphine in clinical practice - a post-marketing surveillance study in 13179 patients. Curr Med Res Opin 2005; 21: 1147-1156.

20 Gallagher A, Leighton S, van Staa T. Utilization characteristics and treatment persistence in patients prescribed low-dose buprenorphine patches in primary care in the United Kingdom: a retrospective cohort study. Clin Ther 2009; 31: 1707-1715. 